\title{
The International Neuroinformatics Coordinating Facility: Evaluating the First Years
}

\author{
Erik De Schutter
}

Published online: 28 July 2009

(C) Humana Press 2009

In January 2004 the ministers of research of the OECD countries endorsed the recommendation from the Neuroinformatics Working Group of the OECD Global Science Forum to start a global neuroinformatics initiative to coordinate international research and resources in the field. As a result, the International Neuroinformatics Coordinating Facility (INCF) was established in August 2005. The INCF has an unusual structure for an international initiative, shown in Fig. 1. It is build on top of national nodes, which are centers of excellence in neuroinformatics supported by national governments and which promote the growth of neuroinformatics at the local level, coordinated by a secretariat. A governing board, composed of representatives from the 15 supporting member countries from Asia, Europe and North America (see http://www.incf.org for an up to date list), provides overview and strategic planning to the activities of the secretariat.

In November 2005, after a competitive bid, the Karolinska Institute in Stockholm, Sweden was selected as the site for the secretariat. Dr. Jan Bjaalie was appointed executive director of the secretariat in 2006, a role taken over by Dr. Mark Ellisman earlier this year.

Erik De Schutter is co-editor in chief of this journal and also member of the INCF governing board and chair of the INCF program for largescale computing.

\footnotetext{
E. De Schutter

Computational Neuroscience Unit,

Okinawa Institute of Science and Technology,

Okinawa, Japan

E. De Schutter $(\square)$

Theoretical Neurobiology, University of Antwerp,

Antwerp, Belgium

e-mail: erik@tnb.ua.ac.be
}

Four years after its foundation the operations of the INCF were reviewed as a prelude towards a decision on extending its life beyond the initial five year period. In this editorial I will present a personal overview of the main activities of the INCF based on a document that was prepared for the international review process, which will be described at the end.

The most established activities of the INCF are its programs, the web portal and the Neuroinformatics Congress. In addition, there are local initiatives managed by the national nodes, that are beyond the scope of this editorial, and a series of workshops on educational activities.

The INCF programs have had a slow start but are now becoming quite visible and it is my belief that these activities will become the main contribution of the INCF to the field. All three active programs have a focus on the establishment of international standards, which is by definition an activity that cannot be done at a national level. Moreover, neuroscience is lagging behind compared to other biological fields in formulating international standards for nomenclature (Bug et al. 2008) or for describing experimental data (Teeters et al. 2008) or models (Cannon et al. 2007; De Schutter 2008).

The first program is on digital atlasing, which has a focus on the rodent brain. Central to this program is a new standard for mapping, called the Waxholm Space (named after the site of a key meeting in 2008). The Waxholm Space is a coordinate-based reference space for the mapping and registration of neuroanatomical data. FIrst steps in its construction are the development of a standardized acquisition procedure for passing data into the Space, using a high-resolution MRI dataset and companion Nissl-stained reconstructions. Next key reference atlases will be registered into the Space and a set of best practices for experimenters to ensure Waxholm Space compatibility will be proposed. 
Fig. 1 The organizational structure of the INCF (C INCF)

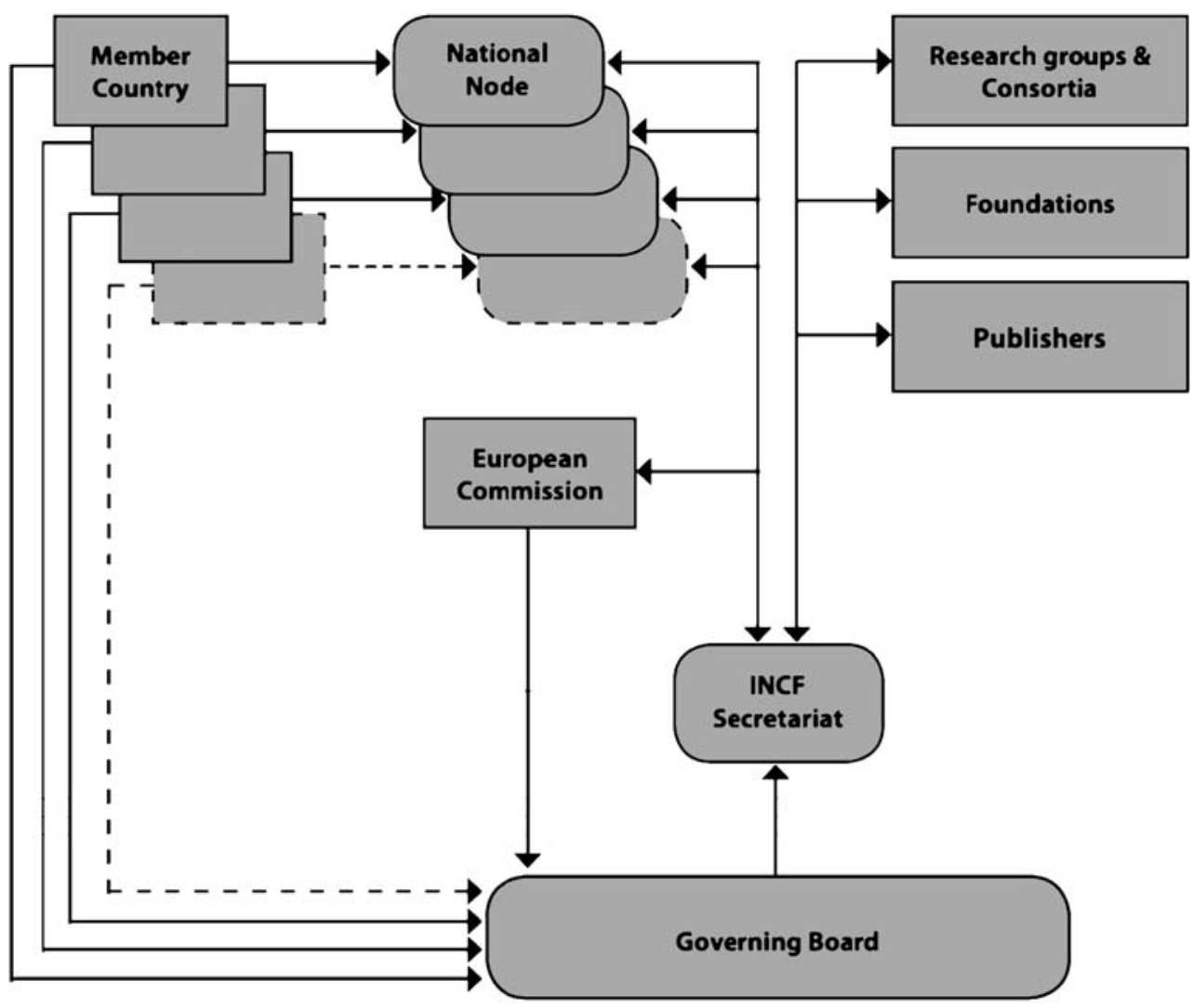

The second program is on ontologies of neural structures (PONS). It will establish a platform for translation and clarification of terminologies in neuroscience, in close interaction with the Neuroscience Information Framework (Gardner et al. 2008). A first product is the structural lexicon, a prototype wiki to gather consistent definitions of structural terms across anatomical scales. This initial wiki will now be further populated with content, with the goal to eventually open it to community input. The structural lexicon, in turn, will form the basis for more formal ontologies for cross species anatomy. In parallel, a neuron registry and a convention for naming neurons are being established, in collaboration with existing initiatives like the Petilla standards for the description of cortical interneurons (Ascoli et al. 2008).

The final program tries to establish standards for model sharing and intercompatibility in large-scale modeling. One major challenge in computational neuroscience is that, because of a wide range of simulation tools being used, it is unlikely that one laboratory can reproduce the results obtained by another group, even if they deposit the model in a database like ModelDB (Cannon et al. 2007). This problem is due to the lack of widely accepted simulatorindependent language standards to communicate models, despite notable attempts (Crook et al. 2007). The systems biology community has led the way in proposing effective solutions to this problem like the SBML and CellML languages (De Schutter 2008), which fostered a strong growth of diverse software tools supporting systems biology and physiome modeling. The INCF program focuses on a fast growing area in computational neuroscience, spiking networks of simple model neurons, as a level of modeling where the initiative is expected to have a large impact. A community-based development effort has begun to establish a machine-readable declarative language standard that describes such network models. In addition this program has supported the development of an API, the MultiSimulation Coordinator (MUSIC), that enables largescale neuron simulators using MPI to internally exchange data during runtime.

As mentioned earlier, the INCF activities were recently reviewed by an international site visit team consisting of K.-P. Hoffman (Germany), M. Ito (Japan), P. Messina (USA), R. Morris (UK) and K.L. Olsen (USA). The conclusions of this team were very positive and they strongly recommended a continuation of the INCF. It specifically lauded the commitment of the host site and the role of the chair of the governing board (Dr. S. Grillner) and of the executive directors in establishing a strong strategic plan where the INCF could act as a coordinator without competing with or duplicating existing initiatives. These recommendations will now be evaluated by the governing board and the member countries. Assuming that the advise to continue the INCF for another five years is 
accepted, important decisions will need to be made on where to orient future new program efforts and how to ensure sufficient funding for these important activities.

\section{References}

Ascoli, G. A., et al. (2008). Petilla terminology: nomenclature of features of GABAergic interneurons of the cerebral cortex. Nature Reviews. Neuroscience, 9, 557-568.

Bug, W. J., Ascoli, G. A., Grethe, J. S., Gupta, A., FennemaNotestine, C., Laird, A. R., et al. (2008). The NIFSTD and BIRNLex vocabularies: building comprehensive ontologies for neuroscience. Neuroinformatics, 6, 175-194.
Cannon, R. C., Gewaltig, M. O., Gleeson, P., Bhalla, U. S., Cornelis, H., Hines, M. L., et al. (2007). Interoperability of neuroscience modeling software: current status and future directions. Neuroinformatics, 5, 127-138.

Crook, S., Gleeson, P., Howell, F., Svitak, J., \& Silver, R. A. (2007). MorphML: level 1 of the NeuroML standards for neuronal morphology data and model specification. Neuroinformatics, 5, 96-104.

De Schutter, E. (2008). Why are computational neuroscience and systems biology so separate? PLoS Computational Biology, 4, e1000078.

Gardner, D., et al. (2008). The neuroscience information framework: a data and knowledge environment for neuroscience. Neuroinformatics, 6, 149-160.

Teeters, J. L., Harris, K. D., Millman, K. J., Olshausen, B. A., \& Sommer, F. T. (2008). Data sharing for computational neuroscience. Neuroinformatics, 6, 47-55. 